\title{
1 Microfluidic Electric Egg-Laying Assay and Application to In-vivo Toxicity 2 Screening of Microplastics using C. elegans
}

3 Khaled Youssef ${ }^{\mathrm{a}}$, Daphne Archonta ${ }^{\mathrm{a}}$, Terrance J. Kubiseseki ${ }^{\mathrm{b}}$, Anurag Tandon ${ }^{\mathrm{c}, \mathrm{d}}$, and Pouya Rezai*a

$4 \quad{ }^{\mathrm{a}}$ Department of Mechanical Engineering, York University, Toronto, ON, Canada

$5 \quad{ }^{\mathrm{b}}$ Department of Biology, York University, Toronto, ON, Canada

$6 \quad{ }^{\mathrm{c}}$ Tanz Centre for Research in Neurodegenerative Diseases, Toronto, Ontario, Canada

$7 \quad{ }^{\mathrm{d}}$ Department of Medicine, University of Toronto, Toronto, Ontario, Canada

8 * Corresponding Author: BRG 433B, 4700 Keele St, Toronto, ON, M3J 1P3, Canada; Tel: 416-736-2100 ext.

9 44703; Email: prezai@yorku.ca

10 Abstract

11 Environmental pollutants like microplastics are posing health concerns on aquatic animals and the ecosystem.

12 Microplastic toxicity studies using C. elegans as a model are evolving but methodologically hindered from

13 obtaining statistically strong data sets, detecting toxicity effects based on microplastics uptake, and correlating

14 physiological and behavioural effects at an individual-worm level. In this paper, we report a novel

15 microfluidic electric egg-laying assay for phenotypical assessment of multiple worms in parallel. The effects

16 of glucose and polystyrene microplastics at various concentrations on the worms' electric egg-laying, length,

17 diameter, and length contraction during exposure to electric signal were studied. The device contained eight

18 parallel worm-dwelling microchannels called electric traps, with equivalent electrical fields, in which the

19 worms were electrically stimulated for egg deposition and fluorescently imaged for assessment of neuronal

20 and microplastic uptake expression. A new bidirectional stimulation technique was developed, and the device

21 design was optimized to achieve a testing efficiency of $91.25 \%$. Exposure of worms to $100 \mathrm{mM}$ glucose

22 resulted in a significant reduction in their egg-laying and size. The effects of $1 \mu \mathrm{m}$ polystyrene microparticles

23 at concentrations of 100 and $1000 \mathrm{mg} / \mathrm{L}$ on the electric egg-laying behaviour, size, and neurodegeneration of

24 N2 and NW1229 (expressing GFP pan-neuronally) worms were also studied. Of the two concentrations, 1000

$25 \mathrm{mg} / \mathrm{L}$ caused severe egg-laying deficiency and growth retardation as well as neurodegeneration. Additionally,

26 using single-worm level phenotyping, we noticed intra-population variability in microplastics uptake and

27 correlation with the above physiological and behavioural phenotypes, which was hidden in the population-

28 averaged results. Taken together, these results suggest the appropriateness of our microfluidic assay for

29 toxicological studies and for assessing the phenotypical heterogeneity in response to microplastics.

30 Keywords: C. elegans, Microfluidics, Egg-laying, Electric field, Glucose, Microplastics. 


\section{INTRODUCTION}

2 The discharge of environmental toxicants such as plastics, pesticides, carcinogens, antimicrobial products, and neurotoxins to the environment is escalating, which poses a significant burden on society, and the ecosystem's

4 health and safety. ${ }^{1-3}$ Some of these toxicants have been linked to neurodegeneration effects and reproductive 5 complications. ${ }^{4,5}$ A total of 359 million metric tons of plastics were manufactured from 1950 to $2018^{6}$, leading 6 to a severe accumulation of plastic waste in the environment. Plastic debris of less than $5 \mathrm{~mm}$ in size, termed 7 microplastics ${ }^{1}$, has become a major concern because it can travel over long distances in the air and aquatic 8 environments while being easily ingested by organisms and animals., ${ }^{3,5,7,8}$

9 The effects of microplastics and their characteristics of shape, size, and concentration on marine life and the 10 ecosystem have been studied and shown to result in various dose-dependent toxicities, including neural, 11 behavioural, and reproductive toxicity. ${ }^{9-12}$ The use of mammalian animal models limits the sample size in 12 these studies and weakens their statistical strength. Such assays are also laborious, expensive, and time13 consuming. ${ }^{13,14}$ Rapid and high-throughput in-vivo screening assays for microplastic toxicity studies are 14 urgently needed. In this front, simple model organisms such as Caenorhabditis elegans, Drosophila melanogaster, and Danio rerio have been used for toxicological studies to rapidly examine the effects of different pollutants at throughputs higher than animal-based assays. ${ }^{15-18}$

17 C. elegans has been used as a model organism for studying microplastics toxicity, offering versatile experimental advantages including small size, short life cycle, ease of maintenance, biological simplicity, and body transparency, leading to its suitability for genetic modification and high-throughput cell-to-behaviour toxicity screening. ${ }^{19-21}$ Ingestion and intestinal accumulation of microplastics smaller than $5 \mu \mathrm{m}$ have been

21 shown in C. elegans. ${ }^{10,22,23}$ Microplastics negatively affected C. elegans phenotypical behaviours such as locomotion and body bend frequency as well as its growth and reproduction. ${ }^{9-12,22-24}$

23 Lei et al. ${ }^{11}$ studied the effects of exposing C. elegans to $1 \mathrm{mg} / \mathrm{L}$ of $0.1-5 \mu \mathrm{m}$ polystyrene microparticles, using 24 locomotory behaviours, growth, and lifespan as toxicity indicators. Their investigations demonstrated that $251 \mu \mathrm{m}$ particles significantly deteriorated the survival and growth rate and caused oxidative damage to cholinergic and GABAnergic neurons, which was ameliorated by natural antioxidants such as curcumin. The same group investigated the toxic effects of different microplastics, including polyamides, polyethylene, polypropylene, polyvinyl chloride, and polystyrene at various concentrations of $0.5-10 \mathrm{mg} / \mathrm{m}^{2}{ }^{12}$ All microplastics showed similar toxic effects on the survival rate of $C$. elegans, indicating no apparent dose- or material-dependent relationship. However, different sizes of 0.1-5 $\mu \mathrm{m}$ microparticles showed a size-dependent

31 lethality with a significant decrease in the reproduction rate and brood size at $1 \mu \mathrm{m}$ microparticles. Yu et al. ${ }^{22}$ 32 investigated the toxicity mechanisms of $1 \mu \mathrm{m}$ polystyrene microplastics on $C$. elegans at different concentrations of 0-100 $\mu \mathrm{g} / \mathrm{L}$. Exposure to concentrations higher than $10 \mu \mathrm{g} / \mathrm{L}$ induced a significant reduction in worms' head thrash, body bend, body length, and brood size. The toxicity was attributed to the increased 
1 reactive oxygen species expression and intestinal damage. The above-mentioned studies showed the effects of

2 microplastics at relatively low concentrations. Therefore, Kim et al. ${ }^{25}$ exposed C. elegans to 42 and $530 \mathrm{~nm}$

3 polystyrene particles at higher concentrations up to $100 \mathrm{mg} / \mathrm{L}$ and showed a significant reduction in the brood

4 size. The effect of microplastics on other behaviours of $C$. elegans, such as reproduction, remains largely

5 unknown. Another gap is the heterogeneity of microplastics uptake by the worms and a lack of understanding

6 of correlating phenotypic toxicities, which requires the use of single-worm assays.

7 C. elegans reproduction is a rhythmic activity that has been established as a robust readout for investigating

8 the toxic effects of different materials. ${ }^{22,26}$ The egg-laying rate is measured by performing progeny counting

9 over several hours. The entire process takes up to 8 hours and requires worm picking expertise without

10 affecting its health, followed by counting the eggs. This conventional technique is cumbersome and limited in

11 throughput to a few worms per hour. To address this limitation, we recently reported a novel technique to

12 stimulate egg-laying of adult C. elegans on-demand using electric pulses in a microchannel (termed electric

13 egg-laying). ${ }^{27,28}$ The throughput of our device was limited to 5 worms/hr, hindering the feasibility of using it

14 in toxicological studies on 100s of worms/assay.

15 In this paper, we report a new microfluidic device for electric egg-laying analysis and on-chip fluorescent 16 imaging of multiple worms in parallel and apply it for the first time to toxicity screening of microplastics. The 17 established effect of glucose on natural reproduction ${ }^{29}$ was used as a proof-of-principle experiment to test the 18 suitability of our assay. Then, we demonstrated the novel application of our method for microplastic toxicity 19 studies, showing the adverse effects of microplastics on the electric egg-laying behaviour of C. elegans and its 20 correlation with microplastic accumulation in the worms. We achieved an assay time of 10 min and a 21 throughput of up to $40 \mathrm{worms} / \mathrm{hr}$, which was mainly limited by our microscopic field of view. Our method is 22 significantly faster than conventional egg-laying assays with $4 \mathrm{hr}$ assay time and $5 \mathrm{worms} / \mathrm{hr}$ throughput. 23 Moreover, we showed another interesting advantage of our device for investigating the effect of microplastics 24 uptake in correlation with multiple physiological and behavioural phenotypes of C. elegans, all at a single25 worm resolution, which is not readily achievable by conventional methods.

\section{MATERIALS AND METHODS}

\section{C. elegans culturing}

28 Wild type $C$. elegans strain was obtained from the Caenorhabditis Genetics Center (University of Minnesota, 29 USA) and maintained at approximately $22^{\circ} \mathrm{C}$ on freshly prepared standard nematode growth media (NGM) 30 plates with Escherichia coli (E. coli) strain $O P 50$ as a food source. ${ }^{30}$ All assays were performed with well-fed 31 gravid hermaphrodite worms (day one post young adult stage $(\sim 64 \mathrm{hrs})$ ) obtained using the conventional 32 alkaline hypochlorite (bleach) treatment. ${ }^{31}$ Briefly, one week prior to the experiments, a chunked NGM plate 33 was prepared and left for three days until a majority of the worms reached the gravid adult stage. All worms 
were washed off the plate in a $15 \mathrm{~mL}$ Eppendorf tube and exposed for $10 \mathrm{~min}$ to a solution of $3.875 \mathrm{~mL}$ double-distilled water, $125 \mu \mathrm{L} \mathrm{NaOH}$, and $1 \mathrm{~mL}$ commercial bleach. The eggs were then collected by washing off the bleach solution using double-distilled water and centrifuging at $1500 \mathrm{rpm}$. The collected eggs were allowed to hatch into $\mathrm{L} 1$ larvae overnight in $1 \mathrm{~mL}$ of $\mathrm{M} 9$ buffer $\left(3 \mathrm{~g} \mathrm{KH}_{2} \mathrm{PO}_{4}, 6 \mathrm{~g} \mathrm{Na}_{2} \mathrm{HPO}_{4}, 5 \mathrm{~g} \mathrm{NaCl}\right.$, and 1

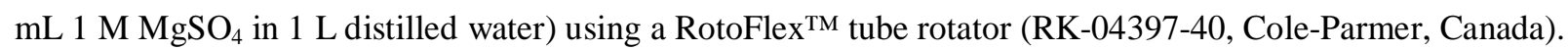

L1 larvae were collected by centrifuging and seeded on top of NGM plates, containing glucose or microplastics when needed.

\section{C. elegans exposure to glucose and microplastics}

9 Glucose was used as an established toxicant to natural egg-laying ${ }^{29}$ to test the performance of our multi-worm 10 device before using microplastics. NGM plates were prepared 5 days before the experiment, either with or 11 without $100 \mathrm{mM}$ of glucose, and left for three days before bacterial seeding. ${ }^{29}$ Luria Broth (LB) media (10 g 12 Bacto-tryptone, $5 \mathrm{~g}$ Bacto-yeast, and $5 \mathrm{~g} \mathrm{NaCl}$ in $1 \mathrm{~L}$ distilled water) was inoculated with a single colony of $13 \mathrm{OP} 50$ and cultured overnight at $37^{\circ} \mathrm{C}$ in a thermal shaker-incubator. ${ }^{30}$ Then, the NGM plates with or without 14 glucose were seeded with $100 \mu \mathrm{L}$ of the freshly prepared bacteria culture and left for two days before being 15 used. L1 larvae were seeded on top of NGM plates with or without glucose for $\sim 64 \mathrm{hr}$ at $22^{\circ} \mathrm{C}$ until they 16 become gravid adults and ready for our experiments.

17 Some studies ${ }^{11,12}$ have shown that the toxicity of microparticles on C. elegans is size-dependent rather than 18 material- or dose-dependent, and $1 \mu \mathrm{m}$ particles have been shown to be an effective size. Our microplastic 19 exposure protocol was based on the method introduced by Schöpfer et al. ${ }^{24}$. One percent (w/v) stock solutions

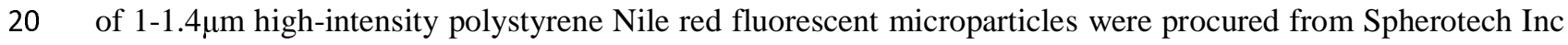
21 (FH-1056-2, Lake Forest, USA). Microplastic feed suspensions were prepared at concentrations of 100 and $221000 \mathrm{mg} / \mathrm{L}$ in a mixture of M9 buffer and E. coli OP50. Aliquots of $100 \mu \mathrm{L}$ of the prepared solutions were seeded on top of bacterial lawns on NGM plates. Then, the microplastic-seeded plates were left to dry for two days before being used to expose the worms. The microplastics distribution was confirmed to be uniform on top of the bacterial lawn with a fluorescent microscope (Leica MZ10F fluorescence microscope, Leica,

26 Wetzlar, Germany). For control experiments, plates were seeded only with $100 \mu \mathrm{L}$ of the E. coli in M9 buffer.

27 The prepared plates were then used for C. elegans growth from the L1 stage to the gravid adult stage ( $64 \mathrm{hr})$ at $22^{\circ} \mathrm{C}$ for our experiments.

\section{Off-chip egg-laying assay}

30 The off-chip egg-laying assay was conducted for the glucose experiments as a validation step to prove that 31 glucose was affecting the worms. ${ }^{29}$ On the day of the experiment, 25 worms per group (control and glucose 32 exposed) were randomly picked and seeded on new NGM plates with and without glucose, respectively. After 33 three hours, the worms were picked off the plates, and the number of laid eggs was counted using an inverted microscope (BIM-500FLD, Bio-imager Inc., Canada) equipped with a camera (SN 14120187, Point Grey 

was compared to the control egg-count.

The microfluidic device shown in Figure 1A was developed to enhance the throughput of our recently reported electric egg-laying method ${ }^{27,28}$, then used for glucose and microplastic toxicity studies.

(A)

(B)

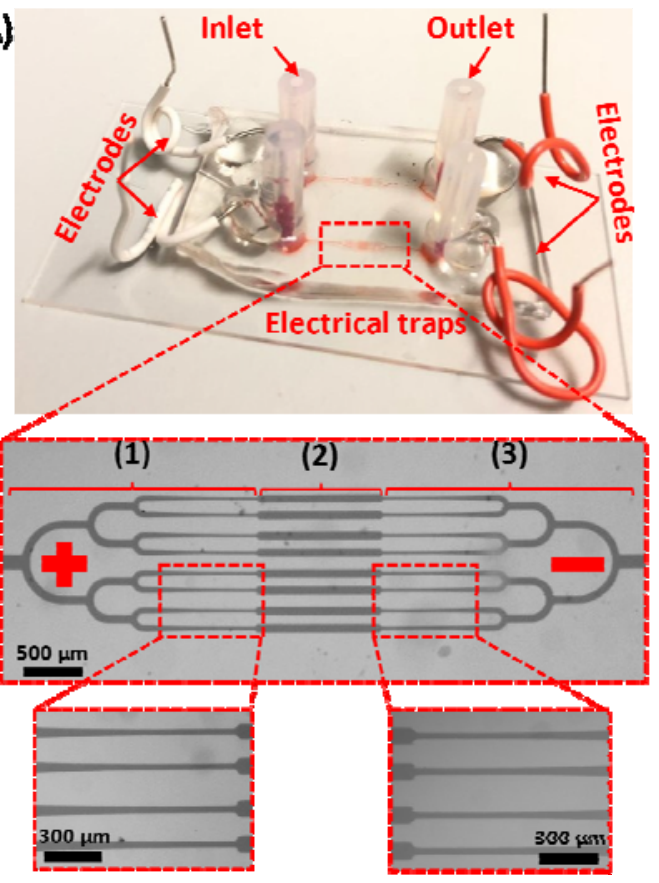

(C)
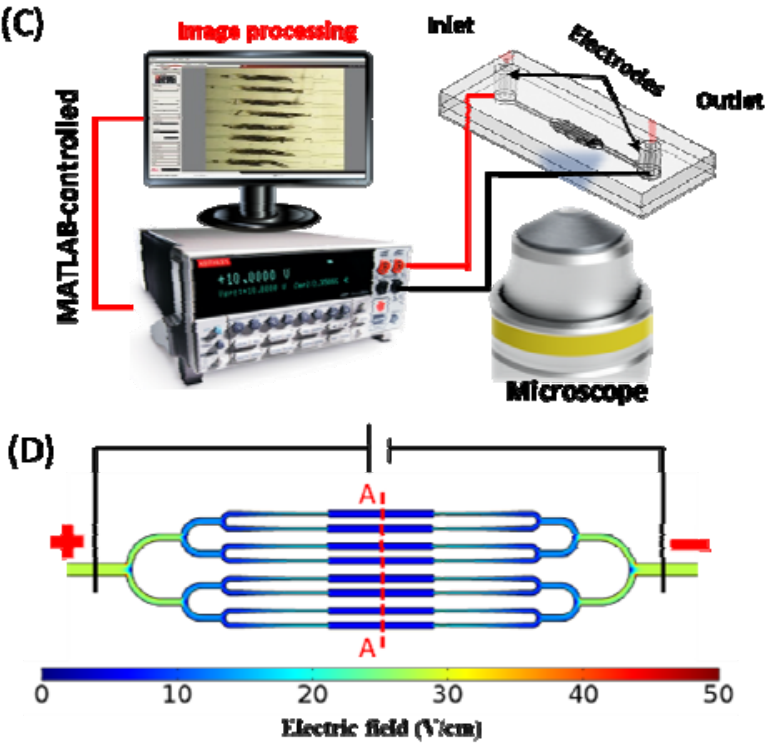

Figure 1: Microfluidic device and experimental setup for studying the electric egg-laying of 8 worms in parallel. (A) An image of the actual chip, housing two devices in parallel, each consisting of one inlet and one outlet interconnected with three-channel sections shown in (B): (1) Tree-like worm loading and distribution channels with end-tapered channels (left inset), (2) 8 parallel electrical traps for worm housing and imaging during the experiment, and (3) worm unloading channels with tapered connections to the electric traps (right inset). (C) Schematic of the experimental setup composing of the microfluidic device mounted on an inverted microscope and connected to a sourcemeter controlled by MATLAB. (D) COMSOL Multiphysics simulation of the electric field distribution throughout the chip to obtain a constant electric field of $6 \mathrm{~V} / \mathrm{cm}$ in the electric traps using V=34 V at the inlet-outlet electrodes.

The $75 \mu \mathrm{m}$ thick polydimethylsiloxane (PDMS) channel network in Figure 1B consisted of three-channel segments, i.e., (1) branching channels for worm loading and distribution with tapered endings (left inset of Figure 1B); (2) eight parallel $85 \mu \mathrm{m}$-wide and $1.3 \mathrm{~mm}$ long electric traps for worms' egg-laying and imaging; and (3) branching channels for worm unloading and collection. The tapered ends of the loading channels 
started from a width of $60 \mu \mathrm{m}$ and narrowed down to different widths of 30, 35, or $40 \mu \mathrm{m}$ in three different

device designs. The electrical traps were located at the mid-section of the device to provide a symmetrical

5 The tree-like loading and unloading channels were inspired by Hulme et al. ${ }^{32}$ to ensure smooth loading and

6 equal distribution of the worms across the 8 channels and maintain equal EF distribution. The loading and EF

7 stimulation techniques benefit from the concept of maintaining constant channel dimensions at each

8 bifurcation to preserve the same pressure and voltage drop up to the electrical traps. The pressure and voltage

9 drops, as well as the hydrodynamic and electrical resistances, can be estimated using Hagen-Poiseuille's (Eq.

10 1) and Ohm's law (Eq. 2), respectively.

$$
\begin{gathered}
\Delta P=R_{f} Q, \quad R_{f}=\frac{128 \mu l}{\pi D^{4}} \\
\Delta V=R_{e} I, \quad R_{e}=\frac{\rho l}{A}
\end{gathered}
$$

11 where $Q$ is the flow rate, $R_{f}$ is the fluid flow resistance, $D$ is the channel hydraulic diameter, $l$ is the channel

12 length, $\mu$ is the fluid dynamic viscosity, $A$ is the cross-sectional area, $R_{e}$ is the electrical resistance, $I$ is the 13 electric current, and $\rho$ is the electric resistivity.

14 Standard photo $^{33}$ - and soft-lithography ${ }^{34}$ techniques were used to fabricate the microfluidic device. At first, a 15 SU-8 master mold was fabricated on a 4 in diameter and $500 \mu \mathrm{m}$ thick silicon wafer (Wafer World Inc., USA) 16 by UV ( $365 \mathrm{~nm}$ with $11.1 \mathrm{~mW} / \mathrm{cm}^{2}$ ) exposure (UV-KUB 2, KLOE, France) of a $75 \mu \mathrm{m}$ thick layer of SU-8 172075 photoresist (MicroChem Corporation, USA) for 20s. Then, to fabricate the negative replica of the master 18 mold, a 10:1 mixture of PDMS elastomer and curing agent (Dow Corning, USA) was degasified, poured over 19 the SU-8 mold, after placing two Masterflex tubes (L/S 14 size, Gelsenkirchen, Germany) over the inlet and 20 outlet reservoirs, and cured for 2 hours at $80^{\circ} \mathrm{C}$. An oxygen plasma machine (PDC-001-HP Harrick Plasma, 21 USA) was used to bond the cured PDMS layer to a glass substrate at $870 \mathrm{mTorr}$ pressure and $30 \mathrm{~W}$ power for 22 30s. Once the device was bonded, the two electrodes were punched through the inlet and outlet tubing for 23 voltage application. Finally, to prevent leakage, the electrode punched areas were sealed with PDMS 24 prepolymer and left to cure at $150^{\circ} \mathrm{C}$ for 5 minutes.

\section{Microfluidic egg-laying assay}

26 Figure 1C depicts the experimental setup used to investigate the electric egg-laying of multiple worms in 27 parallel and simultaneously image them at a single animal resolution. The experimental setup consisted of (1) 28 our microfluidic device installed on a Leica inverted microscope (DMIL LED Inverted Routine Fluorescence 29 Microscope, Leica, Germany) and imaged using a colour camera (MC170 HD, Leica, Germany), and (2) a 30 direct current sourcemeter (Model 2410, Keithley Instruments Inc., USA) connected to the two electrodes at 
1 the inlet and outlet of the microfluidic device. The camera was used for recording movie clips and fluorescent

2 images which were analysed using a custom-made MATLAB code. The code was also used to control the

3 sourcemeter and apply the desired EF pulses while changing the EF polarity when needed. According to our

4 single channel-based egg-laying experiments, an EF of $6 \mathrm{~V} / \mathrm{cm}$ was needed to maximize egg-laying in $C$.

5 elegans. ${ }^{27,35}$ The required voltage to obtain this EF in the new microfluidic device was estimated to be $34 \mathrm{~V}$

6 using a simple COMSOL Multiphysics simulation (Figure 1D), as described in the Supplementary file Section

7 S1.

8 To make our device accessible by the end-users, we eliminated the use of any fluidic control units such as

9 syringe pumps, and the worms were manually manipulated using syringes. Briefly, a syringe filled with M9

10 buffer was connected to the inlet and used to fill in the device. Eight one day old worms were picked up

11 manually from a NGM plate and loaded into the inlet. The worms were pressure-pulsed towards the electrical

12 traps (Figure 2A) (Supplementary Video S1), and the loading efficiency (Eq. 3) was quantified and compared

13 between the three devices with various loading tapered channel sizes.

$$
\text { Loading efficieny }=\frac{\text { Number of occupied traps }}{\text { Total number of traps }} \times 100
$$




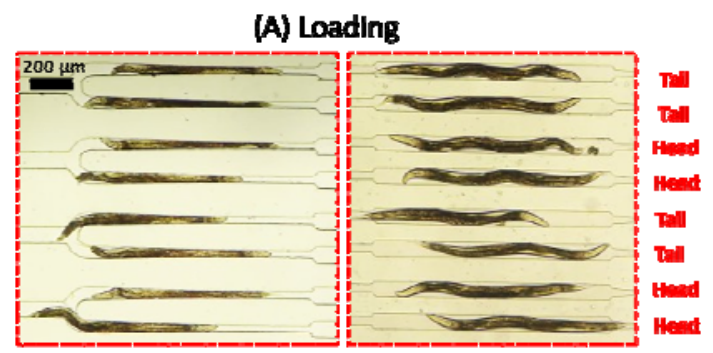

(B) Electric field exposure

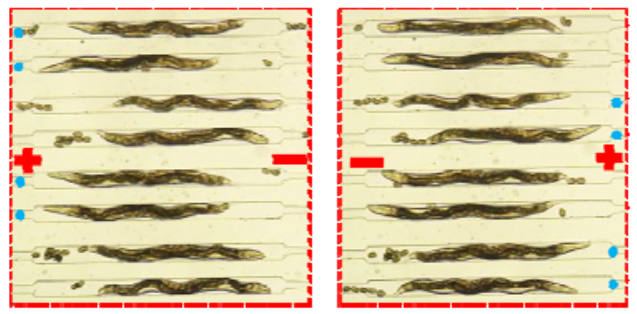

(C) Fluorescent imaging
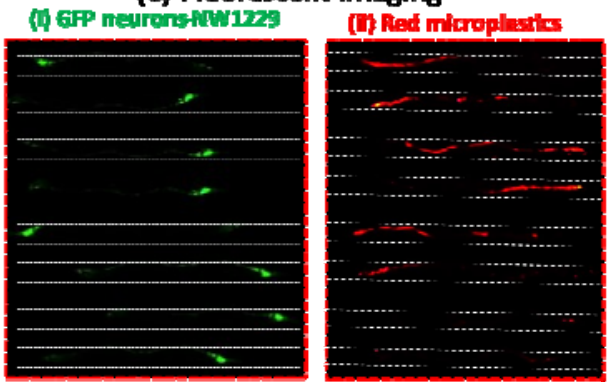

Figure 2: Capturing and investigating the electric egg-laying behaviour and microplastic accumulation in 8 worms in parallel. (A) Loading procedure through the branching channels resulting in a 50\% tail vs. head orientation in the electric traps. (B) Egg-laying response of worms when the anode was at their head or tail. The eggs observed in the traps were deposited mostly when the worms were exposed to anode at their head, as donated by a blue circle beside them. (C) fluorescent images at the traps showing (i) GFP neurons of NW1229 worms and (ii) red fluorescent microplastics ingested by the same worms in (i). Channel walls are highlighted with white dashed lines for better observation.

Prior to EF stimulation, a $60 \mathrm{~s}$ acclimation period was used during which the flow was stopped by maintaining the inlet and outlet tubes at the same height level. Our previous experiments showed that the anode-facing worms could deposit significantly more eggs ${ }^{27,35}$, highlighting that the worm loading orientation with respect to the EF direction could affect our results. To maintain an equal exposure condition among randomly oriented worms in our device (as shown in Figure 2A), we adopted a new EF stimulation technique in which a series of $5 \mathrm{~s}$ anodal and $5 \mathrm{~s}$ cathodal pulses, separated by $25 \mathrm{~s}$ acclimation periods, was applied for $10 \mathrm{~min}$ (Figure 2B). This ensured that each worm was stimulated with 10 anodal and 10 cathodal pulses during an experiment. Next, fluorescent imaging was conducted at $5 \mathrm{x}$ magnification for determining the accumulation of microplastics in each worm that was tested in the device (Figure 2C) (Supplementary Video S1). Then, the worms were flushed out of the chip for the next round of worms to be loaded. 


\section{Data acquisition and analysis}

2 The results, including the electric egg-count, the worms' length, diameter, and length reduction during EF 3 exposure, as well as mean fluorescent intensity expression of neurons (GFP) and microplastics (RFP), were 4 extracted from the recorded videos using our custom-written MATLAB code. ${ }^{27}$ A step was added at the 5 beginning of the code to select the 8 regions of interest around the worms in the electric traps. These regions 6 were cropped, and the worms were individually analyzed, as shown in Figure S1.

7 We used two techniques for presenting our population-based data, i.e., bar plots with the mean \pm standard 8 error of the mean (SEM) and box plots with medians, 25\% and 75\% percentiles, and maximum and minimum 9 data points. The population-based results were reported only for the worms responding to EF. The statistical 10 significance between any two groups was determined using the Mann-Whitney test, while the following 11 representation was used for identifying the significance level, i.e., * for p-value $<0.05$, ** for p-value $<0.01$, $* * *$ for $\mathrm{p}$-value $<0.001$, and $* * * *$ for $\mathrm{p}$-value $<0.0001$.

13 Hierarchical Cluster Analysis (HCA) was performed to understand the toxicity effects of microplastics at 14 single worm resolution. This technique helped identify the worm sub-groups, called clusters, that shared 15 common phenotypes and quantified the differences between individual worms and the sub-groups. The built16 in algorithm in MATLAB was used to perform the clustering analysis. The readout parameters mentioned 17 above were standardized using Eq. 4 with their respective averages from the control worms. Each data point 18 was assigned to a cluster by calculating the minimum Euclidean distance between the data point and the 19 cluster centroid.

$$
X_{\text {std }}=\frac{\left(X_{i}-\bar{X}\right)}{\sigma}
$$

20 where $X_{\text {std }}$ is the standardized value of the data point, $X_{i}$ is the data point of interest (i.e., egg-count, length,

21 diameter, length reduction, and fluorescent intensity), $\bar{X}$ is the control sample mean, and $\sigma$ is the control 22 sample standard deviation.

23 Principal Component Analysis (PCA) was performed to reduce the dimensionality and obtain the parameters

24 with the highest level of information in our datasets. Briefly, in PCA, a new set of variables called the 25 principal components are derived by using linear combinations of the original parameters. The principal 26 components in our studies were calculated using the Minitab software and ranked based on their decreasing 27 eigen-values. Eigen-value represents the amount of variance in the principal component. Therefore, the first 28 two to three principal components can explain $80 \%$ of the total variance, making them suitable for 29 representing the entire dataset, hence reducing the dimensionality. Contributions of each original parameter 30 towards the top principal components were calculated by normalizing the coefficients of the principal 31 components to their $\mathrm{L}^{1}$ norms. The $\mathrm{L}^{1}$ norm is the summation of all absolute values of the coefficient of each 
1 parameter in the principal component. Using this technique, we isolated and reported the original parameters

2 with the highest level of information.

\section{$4 \quad$ RESULTS AND DISCUSSIONS}

5 In this paper, we first investigated three different designs of the microfluidic device (Figure 1) with various

6 tapering channel sizes and quantified the worm loading and orientation efficiencies. Using the best design, we

7 tested a new bidirectional EF stimulation protocol to ensure that the egg-laying results in the multi-worm

8 device were comparable with the results from our single-channel device. As a proof of concept for toxicity

9 analysis, worms were exposed to $100 \mathrm{mM}$ glucose, and their electric egg count was quantified for the first

10 time. Finally, we showed another novel application of our assay for investigating the toxicity effects of

11 polystyrene microplastics and possible correlations between microplastic ingestion level and other on-chip

12 phenotypes at a single-worm resolution.

\section{Loading efficiency of the microfluidic chip}

14 The response of freely moving gravid adult worms to EF in a close-fitting microchannel was studied by us 15 previously at a single worm throughput, and worms were shown to deposit eggs in a controlled manner. ${ }^{27,28}$

16 The effects of EF direction, strength, and exposure duration as well as the worms' age and involvement of 17 neurons and muscles in electric egg-laying were demonstrated, highlighting the potential of this method for 18 toxicological studies at the cell to behaviour level. Here, we improved our technology by performing electric 19 egg-laying and on-chip imaging on 8 worms in parallel, which was restricted by our microscope field of view 20 of $2.2 \times 1.7 \mathrm{~mm}^{2}$. We were also able to keep the worms' identity known during the experiments for correlating 21 the microplastic accumulation levels with the electric egg-laying response and some physiological parameters 22 at a single animal resolution.

23 Our microfluidic device in Figure 1 was designed to (1) distribute 8 worms across the electrical traps, (2) 24 restrain them within traps during EF stimulation, and (3) allow egg release and fluorescent imaging of the 25 worms (Supplementary Video S1). The loading performance of our microfluidic device strongly depended on 26 the tapered channels connecting the inlet channel network to the electrical traps (Figure 1B). They helped 27 impeding the worms from slipping into the electric traps uncontrollably. Three microfluidic devices with 28 tapered loading channels narrowing from a width of $60 \mu \mathrm{m}$ into 30, 35, or $40 \mu \mathrm{m}$ were tested for loading 29 efficiency (Figure S2).

30 A total of 80 worms (in 10 trials) were tested in each device, and the tapered channel with a $30 \mu \mathrm{m}$ wide outlet 31 aided in smooth worm loading into the electrical traps with the highest loading efficiency of $91.25 \%$ (73/80 32 worms). The 35 and $40 \mu \mathrm{m}$-wide tapered channels showed significantly lower loading efficiencies of $56.25 \%$ 
1 (45/80 worms) and 47.5\% (38/80 worms), respectively. They were large for maintaining the worms during

2 loading while sometimes accepting more than one worm per channel. The same loading technique was used

3 previously by Banse et al. ${ }^{36}$ with a similar tapered loading channel dimension of $28 \mu \mathrm{m}$. Thus, the rest of the

4 experiments were conducted using the $30 \mu \mathrm{m}$-wide tapered channel device.

\section{$5 \quad$ Assay time and EF pulsation effects}

6 In our single-worm electric egg-laying experiments ${ }^{27,28}$, we observed a significantly higher egg-count for 7 worms when the anode was positioned at their anterior sides (i.e., anodal exposure). We call this method a 8 unidirectional pulsation since we could choose the anode position with respect to the single worm orientation 9 in this device. In a new set of experiments with our single-worm device in Figure 3A, 20 unidirectional anodal 10 pulses $(6 \mathrm{~V} / \mathrm{cm}, 5 \mathrm{~s}$ on, $25 \mathrm{~s}$ off) were applied within a 10-minute experimental duration to study the egg-laying 11 of $\mathrm{N}=32$ wildtype worms. The number of eggs laid by a worm per minute dropped rapidly in the first 5 12 minutes and plateaued at almost no eggs per worm from minute 5 to minute 10. Figure 3B shows the total 13 number of eggs laid per worm after 5 or 10 minutes in the single-worm device, with no statistically significant 14 difference between the two groups ( $\mathrm{p}$-value>0.05). Within the first 5 minutes, the worms were exposed to 10 15 unidirectional anodal pulses which were selected for the rest of the experiments.

16 In our preliminary experiments with the multi-worm device, we noticed that the worms were oriented 17 randomly with approximately $50 \%$ head or tail towards the trap (Figure $2 \mathrm{~A}$ ). This prevented the use of 18 unidirectional pulses because approximately half of the worms would have been exposed to cathodal pulses, 19 leading to no egg-laying response and waste of animals. Similar longitudinal orientation in the multi-worm 20 device may have been achieved by preconditioning the worms with a longitudinal stimulus (e.g., controlled $21 \mathrm{EF}^{37}$ or flow ${ }^{38}$ ), but this would have added complexity to our procedures.

22 We tested a new bidirectional pulsation method in order to use all the head and tail loaded worms in the multi23 worm device. Accordingly, a series of 10 bidirectional EF pulses (+6V/cm for $5 \mathrm{~s}, 25 \mathrm{~s}$ off, $-6 \mathrm{~V} / \mathrm{cm}$ for $5 \mathrm{~s}, 25 \mathrm{~s}$ 24 off) were applied, aiming to stimulate each worm with an equal number of anodal and cathodal pulses. While 25 this method ensured exposing each worm to 10 anodal pulses as determined in Figure 3A-3B, it also stretched 26 the experimental duration from 5 to 10 minutes.

27 The effect of bidirectional pulsation with 10 anodal and 10 cathodal pulses (within 10 min) was first 28 investigated in the single-worm device (Figure 3C, middle column). For the sake of comparison, the worms' 29 response to 10 unidirectional anodal pulses within $5 \mathrm{~min}$ in the single-worm device was also provided in 30 Figure $3 \mathrm{C}$, left column. As shown, the responses with both methods were statistically similar, proving that the 31 cathodal exposures did not contribute significantly towards the egg-laying response in the bidirectional 32 exposure method. 

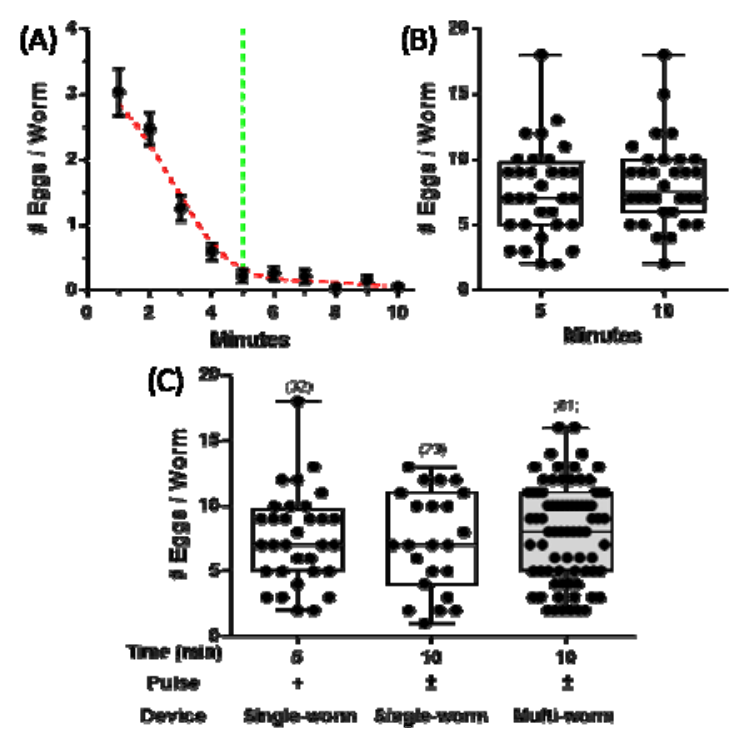

Figure 3: The effects of stimulation time and pulsation method on the electric egg-laying behaviour of adult C. elegans. (A) The number of eggs per worm for $N=32$ worms at $6 \mathrm{~V} / \mathrm{cm}$ using unidirectional pulsation ( $5 \mathrm{~s}$ on, $25 \mathrm{~s}$ off) inside our single-worm device ${ }^{28}$. (B) The total number of eggs deposited at the end of minutes 5 and 10 in (A). (C) Effects of unidirectional $(+)$ pulsation for $N=32$ in the single-worm device and bidirectional ( \pm ) pulsation for $N=23$ and $N=81$ worms in the single- and multi-worm devices, respectively, studied on the number of eggs per worm during 5 and 10 minutes.

The above results ensured us that the bidirectional pulsation technique could be used in the multi-worm device to increase the throughput of the assay. The result of this experiment is also shown in Figure $3 \mathrm{C}$ (right grey column), depicting no statistical difference between the single-worm and multi-worm device responses while enabling us to increase the sample size from 23 to 81 worms and reduce the assay time from 4 to $2 \mathrm{hr}$, respectively.

With the multi-worm device and the bidirectional pulsation method, we were able to reach an egg-laying assay throughput of up to $40 \mathrm{worms} / \mathrm{hr}$, which could be increased in the future with a larger microscope field of view. Compared to our electrical single-worm chip and the natural on-plate egg-laying technique, which can reach a throughput of approximately 5 worms/hr, our multi-worm assay can provide opportunities for testing more chemicals in a faster way. Next, we show the novel applications of our technique for glucose and microplastics toxicity testing.

\section{Effect of glucose on the electric egg-laying of C. elegans}

C. elegans egg-laying circuit consists of a simple neuronal system that is serotonin-controlled. It has been used as an effective readout for identifying the effects of drugs and neurotransmitters. The egg-laying rate is affected by the culture conditions and the availability of food. For instance, different studies have shown the 
1 adverse effects of glucose on the natural egg-laying rate and life span of C. elegans as an application for 2 antidiabetic drug screening. ${ }^{29,39-43}$ Moreover, two recent studies illustrated the associated neurotoxicity effects 3 of glucose on protein aggregation in C. elegans models of Parkinson's and Huntington's diseases. ${ }^{44,45}$ Given 4 the laboriousness and time-consuming nature of these experiments, we asked whether glucose affects the 5 electrical egg-laying response of $C$. elegans and if this method can be used to speed up such chemical 6 screening studies.

7 In each experiment, synchronized one day old adult worms were randomly picked from $100 \mathrm{mM}$ glucose8 dosed and un-dosed control NGM plates and loaded into the multi-worm device for testing using bidirectional 9 pulsation at $\mathrm{EF}=6 \mathrm{~V} / \mathrm{cm}$. Their electric egg-laying count, length, diameter, and body length reduction during 10 EF exposure were quantified, as shown in Figure 4. We also monitored the natural egg-laying behaviour of 25 11 worms off-chip for comparison purposes. Our egg-laying results in Figures 4A and 4B for on- and off-chip 12 worms, respectively, showed that the control worms exhibited a strong egg-laying behaviour as illustrated by 13 their high egg-counts in both experiments. The number of eggs/worm deposited off-chip was higher because 14 eggs were allowed to be laid naturally over $3 \mathrm{hr}$ in this experiment, which was significantly longer than the 10 15 min period used to electrically induce eggs on-chip. In other words, the off-chip worms had more time to 16 reproduce new eggs, while the on-chip worms were egg-depleted rapidly and removed from the device. More 17 importantly, the worms grown on the glucose plates showed noticeable natural and electrical egg-laying 18 deficiencies, determined by their significantly lower egg-counts. Both on- and off-chip egg-laying 19 experiments followed the same trends, indicating the suitability of our microfluidic technique for rapid 20 glucose screening.

21 The off-chip experiment was not only time consuming ( 5 worms $/ \mathrm{hr}$ ) but also prone to the possibility of 22 damaging the worms during the transfer process and miscounting the eggs while searching the plates. 23 Conversely, we performed the on-chip experiments on 90-120 worms in less than three hours (30-40 24 worms/hr throughput) using our multi-worm device with the ability to wash the worms off the plate and into 25 the device without having to pick and potentially damage them. Egg-laying was done on animals spatially 26 restricted in one place, which made the assay less prone to errors in egg counting. Moreover, our technique 27 provided not only the egg-count but also other quantitative readouts, as shown in Figure 4C-4E.

28 It has been shown that glucose affects the worm growth with a discrepancy in the literature, i.e., papers 29 showing an increase $e^{43,45}$ or decrease ${ }^{41,46}$ in the worms' size. Here, we investigated whether exposure to 100 $30 \mathrm{mM}$ glucose results in changes in the worms' growth by measuring their lengths and diameters on the multi31 worm device after the egg-laying assay. As shown in Figure 4C-4D, the glucose-fed worms were significantly 32 shorter (20\%) with no change in their diameter compared to the control worms. Therefore, the electric egg33 laying deficiency in Figure 4B may be attributed to retardation in the growth rate. 

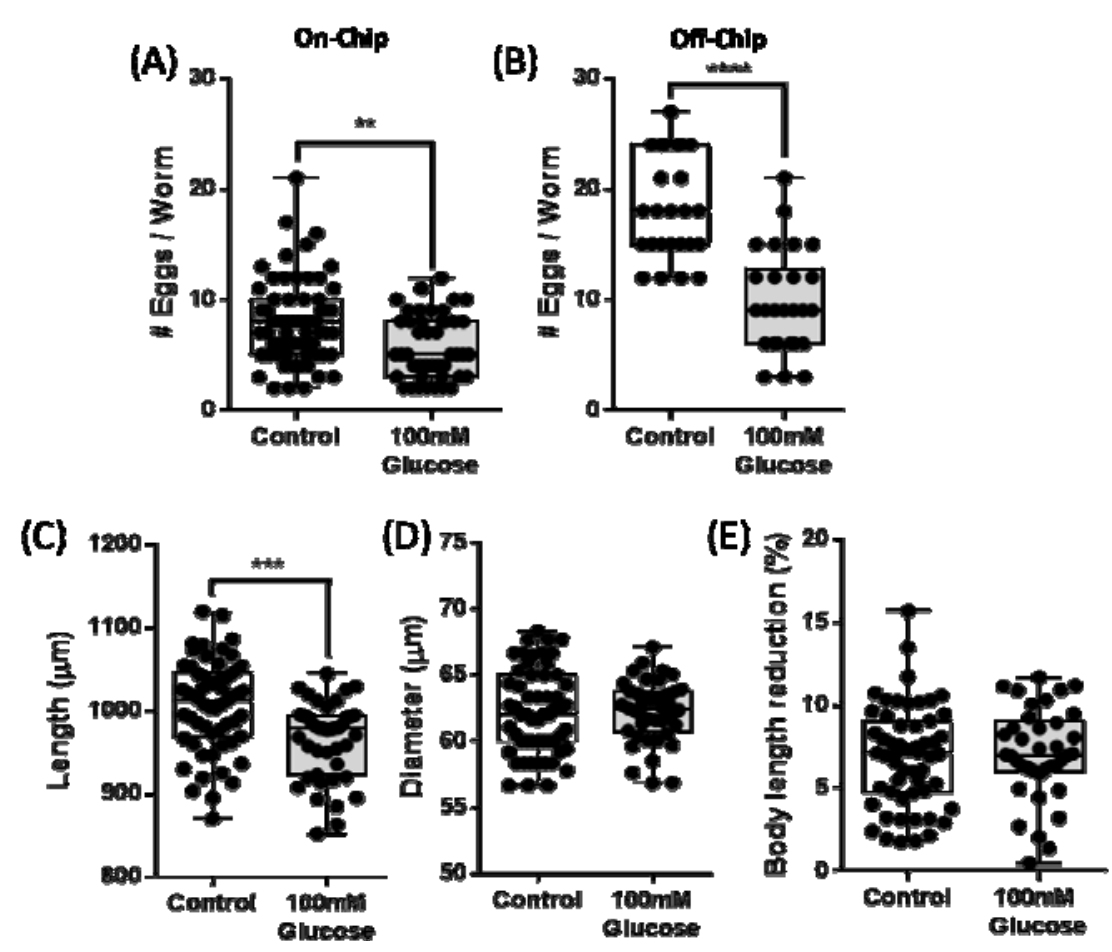

Figure 4: The effect of $100 \mathrm{mM}$ glucose on the $(A)$ natural off-chip $(N=25)$ and $(B)$ electric on-chip $(N=65$ and 44 for control and glucose treated worms respectively) egg-laying of adult C. elegans. EF of 6V/cm was applied for $10 \mathrm{~min}$ in pulses with $\pm 5 \mathrm{~s}$ on and $25 \mathrm{~s}$ off periods. Worms $(C)$ length, $(D)$ diameter, and $(E)$ body

\section{length reduction during anodal stimulation were also quantified.}

The decrease in worms' length might also be accompanied by possible changes in the worms' muscle integrity. In our single-worm egg-laying experiments ${ }^{28}$, we showed that, during EF stimulations, worms contract their bodies in a consistent manner to release eggs. Body shortening was quantified in Figure 4E during the anodal pulses in the multi-worm device for the control and 100mM glucose-fed worms. No significant change was observed in the body length reduction during EF exposure, indicating no detectable effect of glucose on the body wall muscle activities of $C$. elegans. Yet, a deeper analysis of the contraction-relaxation mechanisms might reveal subtle phenotypes that need to be investigated in the future.

Altogether, using glucose, we provided a proof of concept for the use of our technique in determining the toxic effects of chemicals. We also showed that glucose induces abnormal electric egg-laying behaviour in $C$. elegans, which was on par with natural egg-laying behaviour reported earlier by Teshiba et al. ${ }^{29}$. In the future, we aim to use the technique for antidiabetic drug screening and other relevant diseases.

\section{Effect of microplastics on the electric egg-laying of C. elegans}

Recently, exposure to microplastics has been shown to cause significant changes in nematodes locomotory behaviours, life span, and growth rate. ${ }^{23}$ Microfluidics has not been used to study the microplastics toxicity on 
1 C. elegans. Our device not only provides the possibility to obtain various phenotypic behaviours but also

2 allows imaging the worms individually and keeping their identity known during an experiment. In this study,

3 we exposed $\mathrm{N} 2$ worms to 100 and $1000 \mathrm{mg} / \mathrm{L}$ concentrations of $1 \mu \mathrm{m}$ red fluorescent polystyrene

4 microparticles and investigated the accumulation of microplastics in the worms using fluorescent microscopy.

5 The subsequent effects of microplastics on the worms' electric egg-laying, length, diameter, and body

6 contraction during EF exposure were also investigated.

7 Figure 5A shows that increasing the microplastics concentration increased the red fluorescent intensity

8 expression in $\mathrm{N} 2$ C. elegans with microplastics accumulation in the intestine and the pharynx. The

9 quantitative data in Figure 5B, normalized based on the signal at $1000 \mathrm{mg} / \mathrm{L}$, demonstrates that the

10 microplastic uptake at $100 \mathrm{mg} / \mathrm{L}$ was not significant, compared to the control worms, but increased drastically

11 at $1000 \mathrm{mg} / \mathrm{L}$ microplastics.

12 Figure 5C shows that as the microplastics concentration in the culture plate increases, the number of eggs laid

13 electrically by a worm in our device decreases. For instance, at $100 \mathrm{mg} / \mathrm{L}$, the microplastics uptake was

14 relatively low (Figure 5B), resulting in a low toxic effect on egg-laying, confirmed with a slightly lower but

15 statistically insignificant egg deposition of these worms compared to the controls, and a low number of non-

16 responding worms $(\mathrm{N}=2 / 39)$. However, worms exposed to $1000 \mathrm{mg} / \mathrm{L}$ microplastics ingested more

17 microplastics (Figure 5B), and their electric egg-laying was significantly lower than controls $(\mathrm{P}<0.0001$,

18 Figure 5C). It is also worth emphasizing that the number of non-responders ( $N=11 / 51)$ to EF in the 1000

$19 \mathrm{mg} / \mathrm{L}$ sample was significant which confirms the toxicity of microplastics on egg-laying.

20 In terms of body size, Figure 5D-5E show that the worms exposed to $1000 \mathrm{mg} / \mathrm{L}$ microplastics were

21 significantly smaller than the control worms in length $(\mathrm{P}<0.0001)$ and diameter $(\mathrm{P}<0.01)$. However, the

22 worms exposed to $100 \mathrm{mg} / \mathrm{L}$ microplastics were larger than controls in both length and diameter $(\mathrm{P}<0.0001)$.

23 The reason behind this observation is still unknown to us and needs further investigation. Although some

24 researchers have reported a negative effect of microplastics on $C$. elegans length ${ }^{10,11,22}$, others have shown

25 similar results in terms of the non-correlating effect of microplastics on the worms' length. ${ }^{24}$

26 Lastly, Figure 5F shows the length reduction of worms under various microplastic exposure conditions during

27 anodal stimulations (electrical egg-laying) in our device. Length reduction did not change significantly for the

28 treated worms compared to controls $(\mathrm{P}>0.5)$, indicating that microplastics did not affect the muscle

29 contractions. We concluded that the lower egg depositions at higher microplastic concentrations, observed in

30 Figure 5C, were potentially attributed to egg production deficiency and not the muscle activities. 

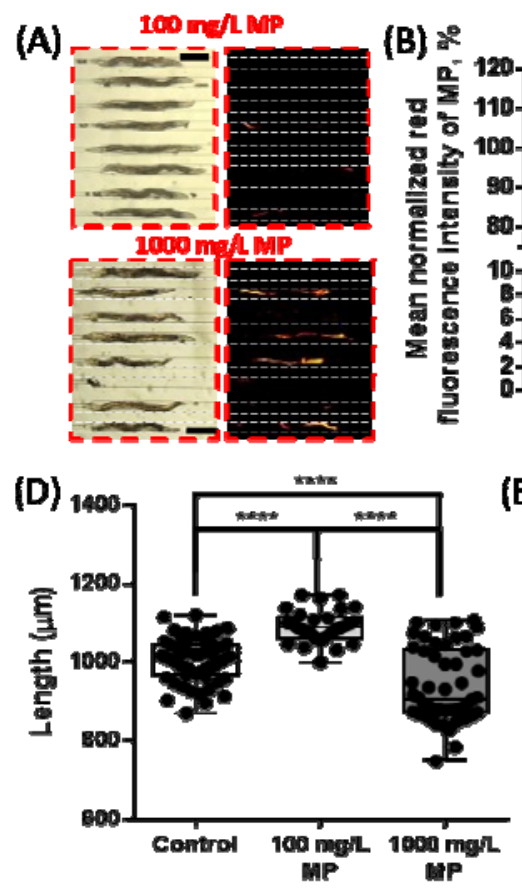

(B) $\mathrm{x}^{\circ}$

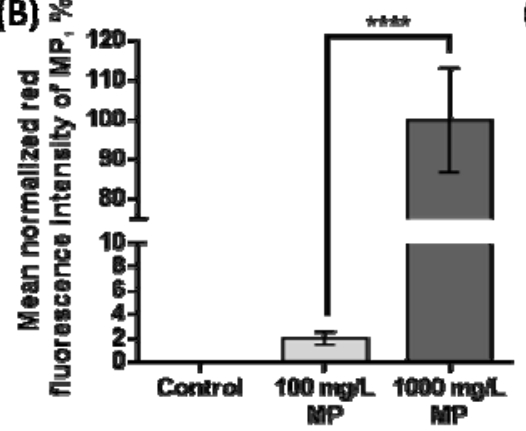

(E)

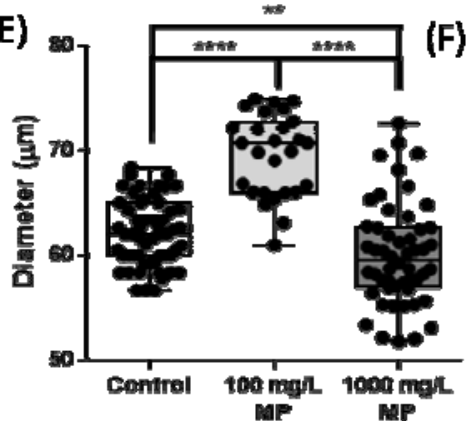

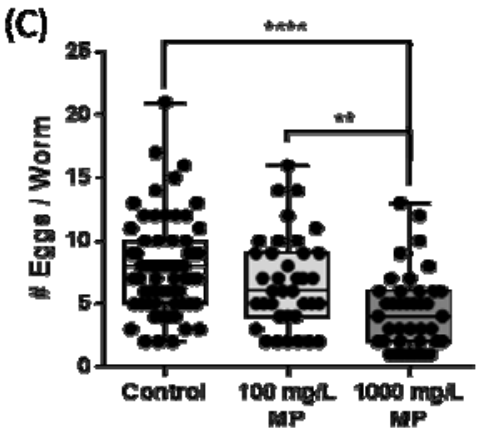

(F)

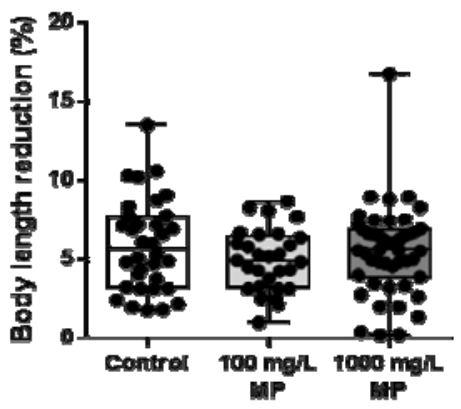

1

Figure 5: The effect of microplastics (MP) at 100 and $1000 \mathrm{mg} / \mathrm{L}$ concentrations on adult $\mathrm{N2}$ C. elegans. $E F=6 \mathrm{~V} / \mathrm{cm}$ was applied for $10 \mathrm{~min}$ in pulses with $\pm 5 \mathrm{~s}$ on and $25 \mathrm{~s}$ off cycles. (A) Bright field and fluorescent images for the worms exposed to $100(N=37 / 39)$ and $1000 \mathrm{mg} / \mathrm{L}(\mathrm{N}=40 / 51)$ microplastics, indicating red fluorescent microplastic uptake (Scale bar $=200 \mu \mathrm{m})$. (B) Microplastic intake rate determined by calculating the mean red fluorescent intensity of the ingested microplastics and normalizing with the signal at $1000 \mathrm{mg} / \mathrm{L}$. (C) Number of electrically deposited eggs per worm counted over $10 \mathrm{~min}$. (D) Length and (E) diameter of the worms at each microplastic concentration compared to control worms $(N=44)$. (F) Maximum total body length reduction during anodal stimulation (electrical egg-laying) in the device.

\section{Effect of microplastics on the neuronal system of C. elegans}

Two recent studies ${ }^{11,47}$ have reported neuronal damages associated with nano- and micro-plastics exposure in C. elegans GABAergic, cholinergic, and dopaminergic neurons, suggesting that the locomotory deficiencies may be due to neurotoxicity. Our goal was to investigate the relationship between the microplastics induced neurotoxicity, growth deficiency, and electric egg-laying using NW1229, a transgenic strain expressing GFP pan-neuronally. NW1229 worms were exposed to 100 and $1000 \mathrm{mg} / \mathrm{L}$ of $1 \mu \mathrm{m}$ red fluorescent polystyrene particles. They were then loaded into the device, and their microplastics intake (red fluorescence), electric egg count, length, diameter, length reduction during anodal stimulation, and GFP expression of the neurons were investigated. The results are shown in Figures 6 and 7. 

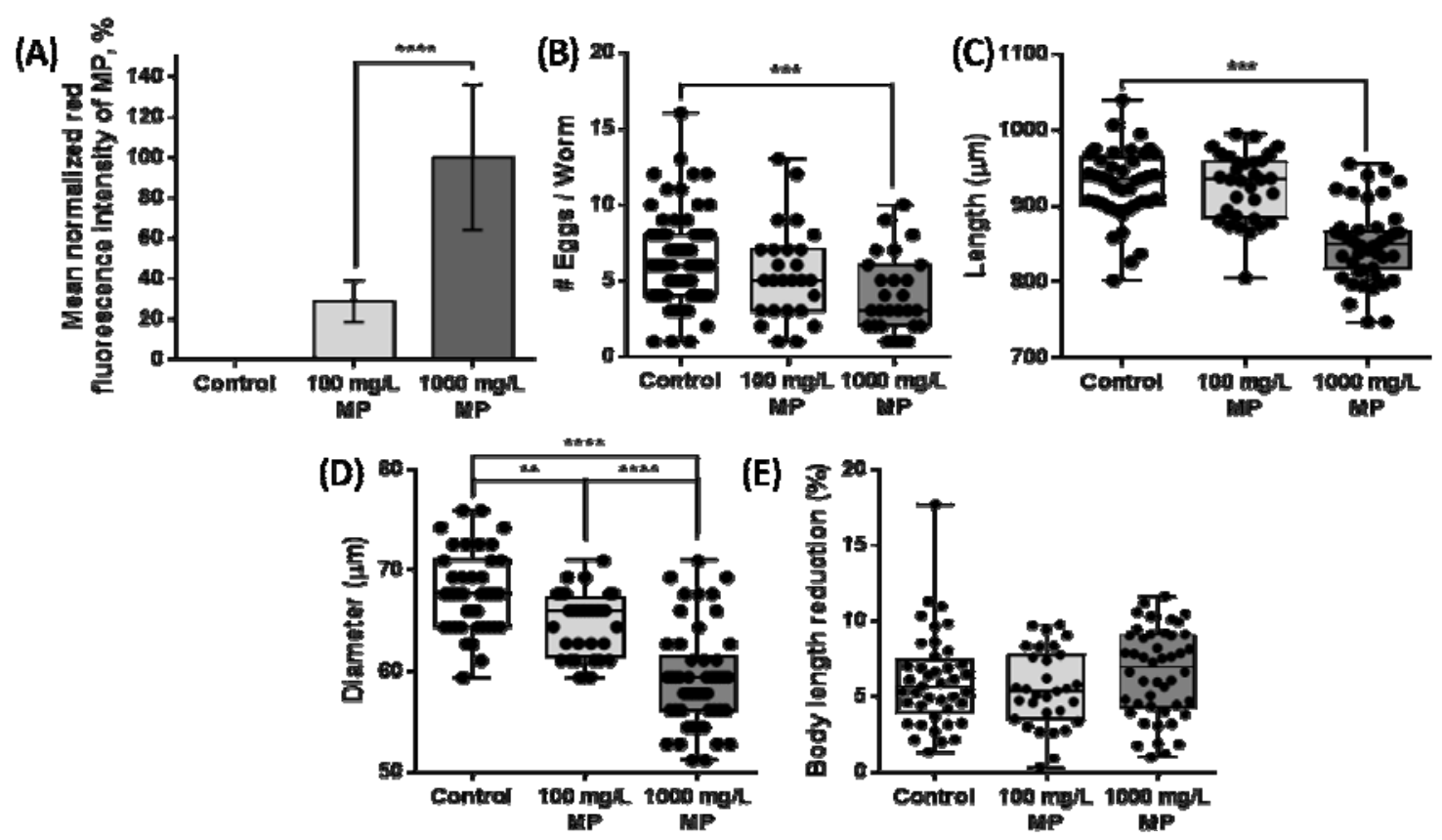

(E)

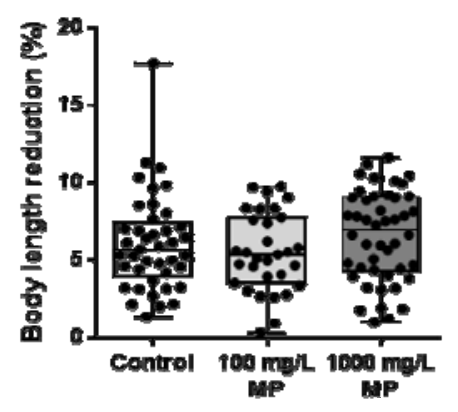

Figure 6: The effect of microplastics $(M P)$ at $100 \mathrm{mg} / \mathrm{L}(\mathrm{N}=26 / 30)$ and $1000 \mathrm{mg} / \mathrm{L}(\mathrm{N}=27 / 45)$ concentrations on adult NW1229 C. elegans expressing GFP pan-neuronally. EF=6V/cm was applied for 10 min in pulses with \pm 5 s on and 25 s off cycles. (A) Microplastic intake rate determined by calculating the mean red fluorescent intensity of the ingested microplastics and normalizing with the signal at $1000 \mathrm{mg} / \mathrm{L}$. (B) Number of electrically deposited eggs per worm counted over $10 \mathrm{~min} .(C)$ Length and $(D)$ diameter of the worms at each microplastic concentration compared to control worms $(N=41)$. (E) Maximum total body length reduction during anodal stimulation (electric egg-laying) in the device.

Results in Figure 6A showed similar trends to the microplastics uptake by N2 worms (Figure 5B). Control worms not exposed to microplastics did not express any red fluorescent while microplastics ingested at 1000 $\mathrm{mg} / \mathrm{L}$ concentration was significantly higher than $100 \mathrm{mg} / \mathrm{L}(\mathrm{P}<0.0001)$. Our egg-laying results in Figure 6B showed that the untreated worms exhibited a strong electric egg-deposition, as demonstrated by their high egg count. However, the worms treated with microplastics exhibited noticeable egg-laying deficiency at 1000 $\mathrm{mg} / \mathrm{L}$ concentration $(\mathrm{P}<0.001)$. At $100 \mathrm{mg} / \mathrm{L}$, microplastics had a slight effect on egg deposition, which was not significantly different from controls, just like what we observed for N2 worms. Also, the number of nonresponders $(N=18 / 45)$ to $E F$ in the $1000 \mathrm{mg} / \mathrm{L}$ sample was higher than the $100 \mathrm{mg} / \mathrm{L}(\mathrm{N}=4 / 30)$. In terms of body size, Figures 6C-6D show that the worms exposed to $100 \mathrm{mg} / \mathrm{L}$ microplastics were similar in length $(\mathrm{P}>$ $0.5)$ but significantly thinner in diameter $(\mathrm{P}<0.01)$ compared to the control worms. This was contrary to what we observed for the N2 strain, which requires further investigation in the future. The worms treated with 1000 $\mathrm{mg} / \mathrm{L}$ microplastics were significantly shorter $(\mathrm{P}<0.0001)$ and thinner $(\mathrm{P}<0.0001)$ than the control worms and the $100 \mathrm{mg} / \mathrm{L}$ treated worms. Finally, we confirmed that the anodal length reductions for all conditions were 

significantly $(\mathrm{P}>0.2)$ altered due to exposure to microplastics (Figure $6 \mathrm{E})$.

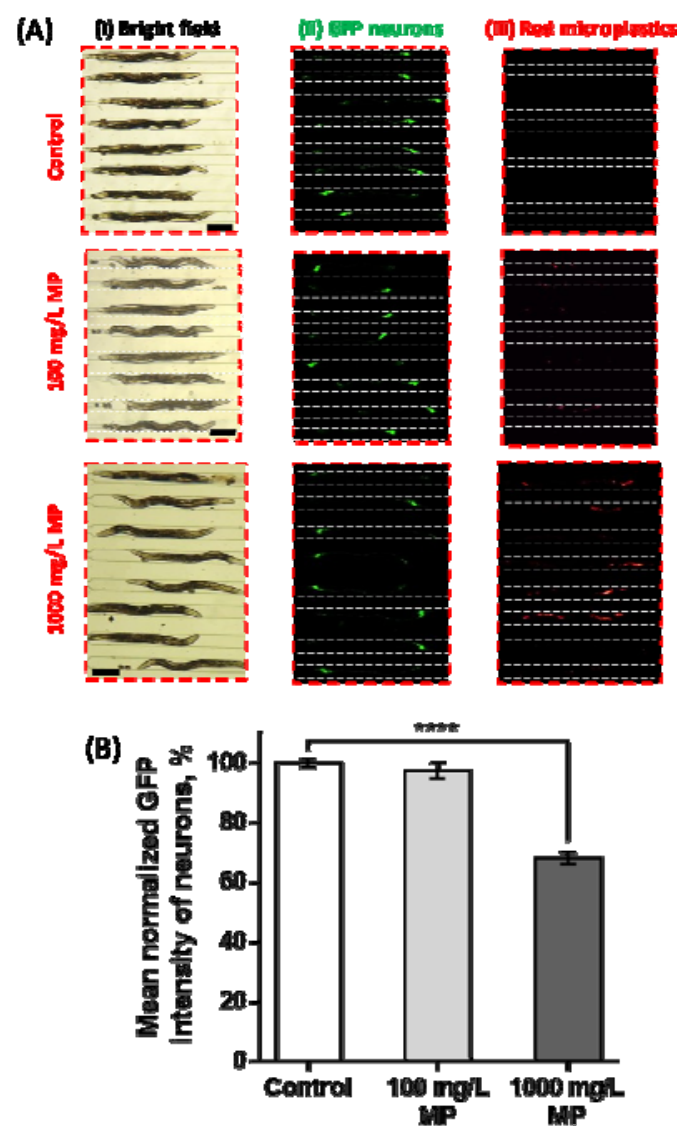

Figure 7: GFP expression of NW1229 C. elegans fed with 100 and $1000 \mathrm{mg} / \mathrm{L}$. (A) Normalized mean fluorescent intensities. $(B)$ Bright field (Scale bar $=200 \mu \mathrm{m})(i)$ and fluorescent images for neuronal system

(ii) and microplastics (iii) at 0 (control), 100, and $1000 \mathrm{mg} / \mathrm{L}$ microplastics.

7 To investigate neurotoxicity, following the $10 \mathrm{~min}$ egg-laying experiment, GFP expressing neurons and red 8 fluorescent microparticles in the worms were separately imaged in the electric traps (Figure 7A). The mean 9 GFP intensities of neurons at both microplastic concentrations were normalized by the average GFP value obtained for the control worms grown without microplastics (Figure 7B). Similar to the electric egg-laying results, exposure to $100 \mathrm{mg} / \mathrm{L}$ microplastics resulted in insignificant $(\mathrm{P}>0.6)$ GFP decay compared to the control worms. However, a significant decrease (P <0.0001) of approximately 35\% in GFP expression was obtained at $1000 \mathrm{mg} / \mathrm{L}$ microplastic exposure (Figure 7B), similar to the egg-count reduction in the same condition (Figure 6B). The decreasing trend in GFP expression at both concentrations is in line with the increasing trend in microplastics uptake, shown in Figure 6A for the NW1229 worms. The above results show the appropriateness of our technique for toxicity assessment from neuron to behaviour level. Moreover, our 
1 results confirmed that microplastics toxicity is not specific towards certain neurons, whereas GFP expression

2 decayed over the entire body of the worm. This was also confirmed previously using off-chip assays that

3 showed neurodegeneration for specific neurons as well as defective locomotory behaviour. ${ }^{23}$

4 Altogether, our results showed the suitability of our microfluidic technique to study glucose and microplastics

5 toxicity in $C$. elegans and detect the associated behavioural and neuronal abnormalities, quantitively and at a

6 population resolution. The following section will show the potential of our device for use in single-worm level

7 neurobehavioural toxicity studies.

8 Single-worm analysis reveals heterogeneity in microplastics uptake and correlating egg-laying and

9 physiological phenotypes

10 In recent years, physicians have found that drug-patient interactions are highly important, demonstrating the

11 need for developing individualized diagnoses and treatments that take patient variabilities into account ${ }^{48}$. In

12 the case of $C$. elegans, multiple microfluidic devices have recently shown that analysing the data in an

13 individual-based manner would reveal subtle phenotypes that were hidden in population measurements ${ }^{49,50}$.

14 Here, we questioned the heterogeneity of microplastics uptake and toxicity, and show that our microfluidic

15 device can be used to perform phenotypical and neuronal analyses at single-animal resolution and at a

16 throughput higher than conventional assays for microplastics toxicity assessment.

17 As described in the Materials and Methods section, HCA and PCA were performed on individual N2 and

18 NW1229 worms treated with microplastics at 100 and $1000 \mathrm{mg} / \mathrm{L}$, and the results were compared with their

19 counterpart control worms. The egg-count, worm length, worm diameter, length reduction during anodal

20 stimulation, and normalized mean GFP and RFP fluorescent intensities, representing neurons and

21 microplastics, respectively, were quantified for individual worms.

22 Figure 8Ai shows the heatmap representing the clustering generated by the HCA algorithm for N2 worms.

23 Columns and rows represent individual worms and their phenotypes, respectively. The data were standardized

24 using Eq.4 based on the control worm parameters. Thus, in Figure 8Ai, the color gradients depict whether a

25 parameter of interest is quantitatively similar (white), lower (dark blue), or higher (dark red) than the average

26 of the control group. The only exemption was the microplastic uptake RFP parameter for which the $100 \mathrm{mg} / \mathrm{L}$

27 data was selected as standardization reference since the control worms were not exposed to microplastics,

28 expressing zero RFP intensity. 

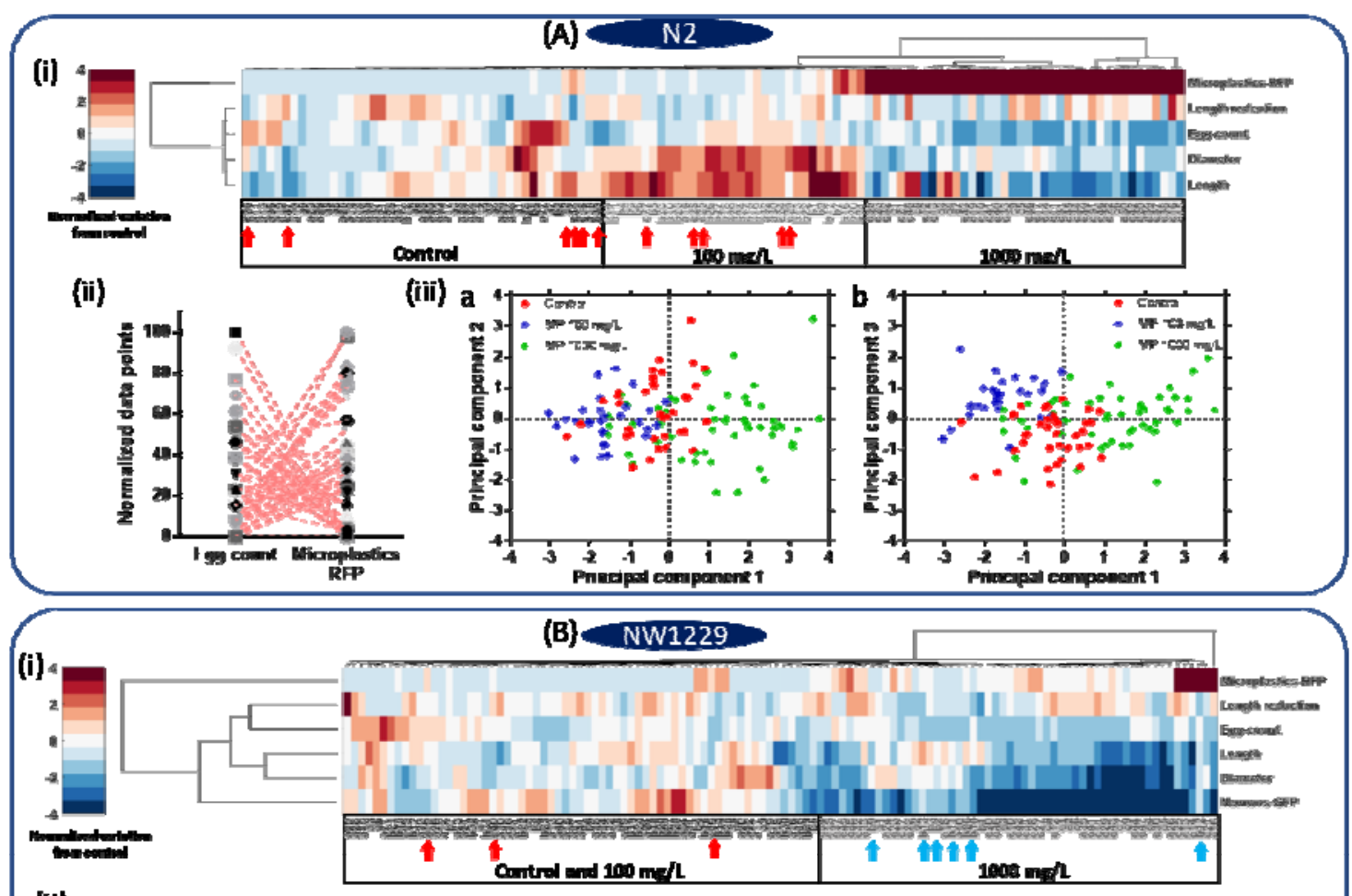

1
(III)

(ii)

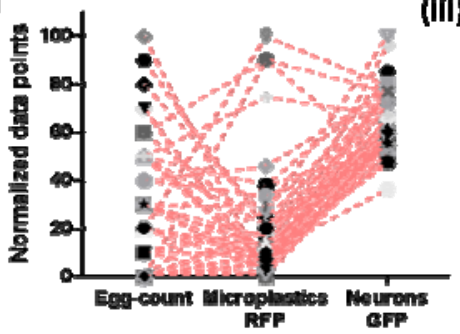

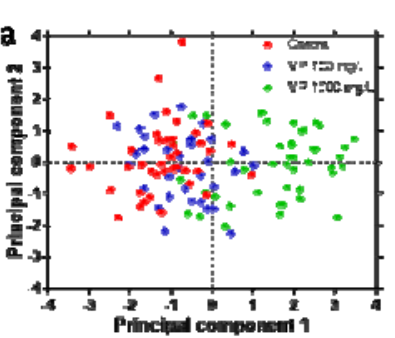

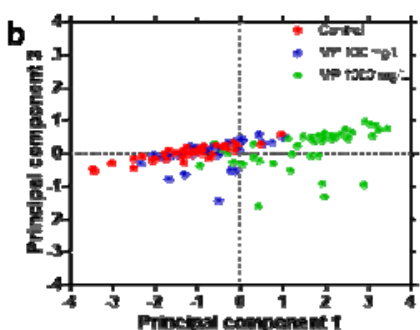

Figure 8: Phenotypic analysis of individual worms, (A) N2 and (B) NW1229, including control worms and ones treated with 100 and 1000 mg/L microplastics. (i) Hierarchical Cluster Analysis (HCA) of different phenotypes of the control and microplastic-exposed worms. (ii) Normalized individual responses of egg-count, microplastic uptake and GFP expression (NW1229 strain only) at $1000 \mathrm{mg} / \mathrm{L}$. (iii) Principal Component Analysis (PCA) of control, 100 and $1000 \mathrm{mg} / \mathrm{L}$ microplastic-exposed worms.

Figure 8Ai shows that the control worms were mostly clustered at the left-hand side of the graph, followed by the worms exposed to 100 and $1000 \mathrm{mg} / \mathrm{L}$ microplastics. Generally, the graph confirms the population trends discussed earlier in the paper, but at an individual worm level. It shows that the microplastics uptake at 1000 $\mathrm{mg} / \mathrm{L}$ was much higher than the $100 \mathrm{mg} / \mathrm{L}$, for most of the worms, and the worms' diameter and length at 1000 $\mathrm{mg} / \mathrm{L}$ were lower than the control and $100 \mathrm{mg} / \mathrm{L}$ worms. Moreover, the electric egg-laying rate for the worms that took more microplastics was always lowered. Interestingly, using the clustering technique, we were able to isolate individual worms treated with $1000 \mathrm{mg} / \mathrm{L}$ microplastics that were behaving like untreated worms, with high egg count and low microplastics accumulation. These worms were clustered within the control or 
$1100 \mathrm{mg} / \mathrm{L}$ populations as depicted with red arrows in Figure 8Ai. This showed the heterogeneity of

2 microplastic uptake in the worms treated in the same way, which necessitates single-animal resolution assays

3 like the one offered here to isolate such worms and understand their correlating phenotypes correctly. Such

4 data points were hidden in our population-based averaging results offered in the previous sections, and this is

5 the case in conventional worm screening assays, especially when they involve behavioral phenotyping.

6 HCA and PCA revealed some hidden correlations between the independent parameters. For instance, using

7 PCA, the contribution of each parameter to the first, second, and third principal components, computed in

8 Table S1, showed that the egg-count and the microplastics uptake level are inversely proportional. In other 9 words, worms with higher microplastics expression were defective in egg-laying. To better visualize this 10 correlation, we focused on the $1000 \mathrm{mg} / \mathrm{L}$ treated worms and normalized the egg-count and microplastics 11 fluorescent expression parameters to obtain similar scales for comparison purposes. Then we plotted both 12 parameters while preserving the identity of individual worms by connecting dash-lines, as shown in Figure 13 8Aii. Moreover, our results were further confirmed by plotting the calculated scores from the PCA using the 14 first, second, and third principal components (Figure 8Aiii). This figure also confirms that the $1000 \mathrm{mg} / \mathrm{L}$ 15 treated worms were clustered together with some individuals behaving like control and existing within the control cluster as discussed above.

17 Similar results were obtained for the NW1229 strain as shown in Figure 8B. Briefly, HCA analysis in Figure $188 \mathrm{Bi}$ created two main clusters for the $1000 \mathrm{mg} / \mathrm{L}$ worms at the right-hand side and the control and $100 \mathrm{mg} / \mathrm{L}$ worms mixed at the left-hand side, explaining their population trends described earlier. The heatmap

20 illustrates increase in the microplastic uptake at $1000 \mathrm{mg} / \mathrm{L}$ exposure, and corresponding decreases in the egg21 count, length, diameter, and neurons GFP expression of the worms. The graph also shows that there was a 22 large variation in microplastics uptake within the $1000 \mathrm{mg} / \mathrm{L}$ population with some worms showing a 23 significantly higher uptake than the others. Moreover, some NW1229 worms, exposed to $100 \mathrm{mg} / \mathrm{L}$ (blue 24 arrows) and $1000 \mathrm{mg} / \mathrm{L}$ (red arrows) microplastics, were not placed in their respective clusters in Figure 8Bi. 25 For these worms, the level of microplastics uptake (or GFP expression) was on par with the same phenotypes 26 in their clusters.

27 The PCA analysis in Table S2 for NW1229 worms showed similar trends to the N2 strain, demonstrating that 28 the microplastics uptake and egg-count were inversely proportional. However, there was no obvious pattern 29 between the microplastics uptake and neurodegeneration. This was observed in Figure 8Bii by plotting the 30 individual responses of $1000 \mathrm{mg} / \mathrm{L}$ worms for the egg-count, microplastics uptake, and neurons fluorescent 31 expression while preserving the worms' identity. Lastly, Figure 8Biii shows that only using the first and 32 second principal components, our results were further confirmed, and the $1000 \mathrm{mg} / \mathrm{L}$ treated worms were 33 clustered together, whereas the control and $100 \mathrm{mg} / \mathrm{L}$ treated worms were mixed. 
1 All in all, we envision that our microfluidic device, coupled with the clustering techniques above, would be of

2 benefit to develop individualized drug and pollutant screening assays for C. elegans.

\section{Conclusion}

4 As an emerging toxicant, microplastics have been recently spotted in abundance throughout the environment.

5 C. elegans is a simple and easy to use model organism for toxicological studies. Previous studies showed the

6 negative effects of microplastics on C. elegans phenotypical behaviours using conventional assays, which are 7 time-consuming and laborious, limiting the possibility of developing high throughput toxicity screening 8 methods. Moreover, previous studies have solely focused on the natural behaviours of $C$. elegans while 9 paying less attention to the sensory-motor evoked phenotypes to assess the neuronal system integrity. 10 Therefore, in this paper, we developed a microfluidic device to exploit the newly introduced C. elegans 11 electric egg-laying behaviour for toxicity studies.

12 Our device enabled trapping and exposing up to 8 individual worms in parallel to EF while allowing on-chip 13 fluorescent imaging. We optimized our microfluidic device and adopted a new bidirectional stimulation 14 technique to achieve a throughput of 40 worms $/ \mathrm{hr}$, which can be further enhanced in the future with a larger 15 microscope field of view. Large datasets of multiple parameters such as electric egg-count, worm length, 16 worm diameter, length reduction, and neuronal and microplastics mean fluorescent intensities were obtained 17 and analyzed in population-averaged and individual-worm approaches.

18 The effect of glucose was investigated as a proof of concept for toxicity assays. Our findings implied that 100 $19 \mathrm{mM}$ of glucose induced abnormal electric egg-laying behaviour and resulted in a smaller body size. Thus, in 20 the future, we aim to use the technique for antidiabetic drug screening. Moreover, we employed our device 21 with two $C$. elegans strains (N2 and NW1229) to investigate the toxicity effects of $1 \mu \mathrm{m}$ polystyrene 22 microparticles at concentrations of 100 and $1000 \mathrm{mg} / \mathrm{L}$ on electric egg-laying and worm size as well as 23 neurodegeneration in a faster manner using single-worm phenotyping. Our results showed that exposing the 24 worms to $1000 \mathrm{mg} / \mathrm{L}$ caused severe egg-laying deficiency and neurodegeneration, and reduction in body size. 25 Lastly, using HCA and PCA, we showed that single-worm phenotyping could reveal heterogeneity in 26 microplastics uptake, which was correlated with the deficiency in egg-laying.

27 In the future, we aim to develop a microfluidic device for life-long phenotyping of microplastics toxicity 28 while allowing for sensory-motor behavioural studies. Our technique can also be used as an ecotoxicity 29 screening technique for determining the sublethal effects of other materials such as heavy metals on $C$. 30 elegans.

\section{Author Contributions}


1 Khaled Youssef: Methodology, Investigation, Formal analysis, Validation, Data curation, Visualization,

2 Writing - original draft. Daphne Archonta: Data curation and analysis. Terrance J. Kubiseski:

3 Conceptualization, Writing - review \& editing. Anurag Tandon: Supervision, Validation, Writing - review \&

4 editing. Pouya Rezai: Conceptualization, Methodology, Validation, Resources, Writing - review \& editing,

5 Supervision, Funding acquisition.

6 Conflicts of interest

7 The author(s) declared no conflict of interest.

\section{Acknowledgements}

9 This work was supported by Natural Sciences and Engineering Research Council (NSERC) of Canada 10 and the Early Researcher Award to PR and the Ontario Trillium Scholarship to KY.

\section{References}

12

13 1. Wright SL, Kelly FJ. Plastic and Human Health: A Micro Issue? Environmental Science and Technology. 2017;51(12):6634-6647. doi:10.1021/acs.est.7b00423

2. Geyer R, Jambeck JR, Law KL. Production, use, and fate of all plastics ever made. Science Advances. 2017;3(7):e1700782. doi:10.1126/sciadv.1700782

17 3. Sharma S, Chatterjee S. Microplastic pollution, a threat to marine ecosystem and human health: a short review. Environmental Science and Pollution Research. 2017;24(27):21530-21547. doi:10.1007/s11356-017-9910-8 microplastics. Proceedings of the National Academy of Sciences. 2016;113(9):2430 LP - 2435. doi:10.1073/pnas.1519019113

5. Prüst M, Meijer J, Westerink RHS. The plastic brain: neurotoxicity of micro- and nanoplastics. Particle and Fibre Toxicology. 2020;17(1):24. doi:10.1186/s12989-020-00358-y

6. PlasticsEurope (PEMRG). (September 25, 2019). Production of plastics worldwide from 1950 to 2018 (in million metric tons)* [Graph]. In Statista. Retrieved December 09, 2020, from https://www.statista.com/statistics/282732/global-production-of-plastics-sin. de Sá LC, Oliveira M, Ribeiro F, Rocha TL, Futter MN. Studies of the effects of microplastics on aquatic organisms: What do we know and where should we focus our efforts in the future? Science of the Total Environment. 2018;645:1029-1039. doi:10.1016/j.scitotenv.2018.07.207

31 8. Pennino MG, Bachiller E, Lloret-Lloret E, et al. Ingestion of microplastics and occurrence of parasite association in Mediterranean anchovy and sardine. Marine Pollution Bulletin. 2020;158:111399. 
doi:10.1016/j.marpolbul.2020.111399

9. Kim SW, Kim D, Jeong SW, An YJ. Size-dependent effects of polystyrene plastic particles on the nematode Caenorhabditis elegans as related to soil physicochemical properties. Environmental Pollution. 2020;258:113740. doi:10.1016/j.envpol.2019.113740

10. Shang X, Lu J, Feng C, et al. Microplastic (1 and $5 \mu \mathrm{m})$ exposure disturbs lifespan and intestine function in the nematode Caenorhabditis elegans. Science of the Total Environment. 2020;705:135837. doi:10.1016/j.scitotenv.2019.135837

11. Lei L, Liu M, Song Y, et al. Polystyrene (nano)microplastics cause size-dependent neurotoxicity, oxidative damage and other adverse effects in Caenorhabditis elegans. Environmental Science: Nano. 2018;5(8):2009-2020. doi:10.1039/c8en00412a

12. Lei L, Wu S, Lu S, et al. Microplastic particles cause intestinal damage and other adverse effects in zebrafish Danio rerio and nematode Caenorhabditis elegans. Science of the Total Environment. 2018;619-620:1-8. doi:10.1016/j.scitotenv.2017.11.103

13. Olivier K, Karanth S. Toxicology testing: in vivo mammalian models. In: An Introduction to Interdisciplinary Toxicology. Elsevier; 2020:487-506. doi:10.1016/b978-0-12-813602-7.00035-1

14. Yong CQY, Valiyaveetill S, Tang BL. Toxicity of Microplastics and Nanoplastics in Mammalian Systems. International Journal of Environmental Research and Public Health. 2020;17(5):1509. doi:10.3390/ijerph17051509

15. Hunt PR. The C. elegans model in toxicity testing. Journal of Applied Toxicology. 2017;37(1):50-59. doi:10.1002/jat.3357

16. Rand MD, Montgomery SL, Prince L, Vorojeikina D. Developmental Toxicity Assays Using the Drosophila Model. Current Protocols in Toxicology. 2014;59(1):1.12.1-1.12.20. doi:10.1002/0471140856.tx0112s59

17. Rubinstein AL. Zebrafish assays for drug toxicity screening. Expert Opinion on Drug Metabolism and Toxicology. 2006;2(2):231-240. doi:10.1517/17425255.2.2.231

18. Caballero MV, Candiracci M. Zebrafish as screening model for detecting toxicity and drugs efficacy. Journal of Unexplored Medical Data. 2018;3(2):4. doi:10.20517/2572-8180.2017.15

19. Youssef K, Bayat P, Peimani AR, Dibaji S, Rezai P. Miniaturized Sensors and Actuators for Biological Studies on Small Model Organisms of Disease. In: Environmental, Chemical and Medical Sensors. Springer; 2018:199-225. doi:10.1007/978-981-10-7751-7_9

20. Youssef K, Tandon A, Rezai P. Studying Parkinson's disease using Caenorhabditis elegans models in microfluidic devices. Integrative biology $\square$ : quantitative biosciences from nano to macro. 2019;11(5):186-207. doi:10.1093/intbio/zyz017

21. Gupta BP, Rezai P. Microfluidic approaches for manipulating, imaging, and screening C. elegans. Micromachines. 2016;7(7):123. doi:10.3390/mi7070123

22. Yu Y, Chen H, Hua X, et al. Polystyrene microplastics (PS-MPs) toxicity induced oxidative stress and 
intestinal injury in nematode Caenorhabditis elegans. Science of the Total Environment. 2020;726:138679. doi:10.1016/j.scitotenv.2020.138679

23. Hu J, Li X, Lei L, Cao C, Wang D, He D. The Toxicity of (Nano)Microplastics on C. elegans and Its Mechanisms. In: He D, Luo Y, eds. Microplastics in Terrestrial Environments: Emerging Contaminants and Major Challenges. Cham: Springer International Publishing; 2020:259-278. doi:10.1007/698_2020_452

24. Schöpfer L, Menzel R, Schnepf U, et al. Microplastics Effects on Reproduction and Body Length of the Soil-Dwelling Nematode Caenorhabditis elegans. Frontiers in Environmental Science. 2020;8:41. doi:10.3389/fenvs.2020.00041

25. Kim Y, Jeong J, Lee S, Choi I, Choi J. Identification of adverse outcome pathway related to highdensity polyethylene microplastics exposure: Caenorhabditis elegans transcription factor RNAi screening and zebrafish study. Journal of Hazardous Materials. 2020;388:121725. doi:10.1016/j.jhazmat.2019.121725

26. Maulik M, Mitra S, Bult-Ito A, Taylor BE, Vayndorf EM. Behavioral phenotyping and pathological indicators of Parkinson's disease in C. elegans models. Frontiers in Genetics. 2017;8(JUN):77. doi:10.3389/fgene.2017.00077

27. Youssef K, Archonta D, Kubiseski TJ, Tandon A, Rezai P. Electric Egg-Laying: Effect of Electric Field in a Microchannel on C. elegans Egg-Laying Behavior. bioRxiv. 2020.

28. Youssef K, Archonta D, Kubiseski TJ, Tandon A, Rezai P. Electric Egg-Laying: Effect of Electric Field in a Microchannel on C. elegans Egg-Laying Behavior. Lab on a Chip. 2021:Under review.

29. Teshiba E, Miyahara K, Takeya H. Glucose-induced abnormal egg-laying rate in Caenorhabditis elegans. Bioscience, Biotechnology, and Biochemistry. 2016;80(7):1436-1439. doi:10.1080/09168451.2016.1158634

30. Stiernagle T. Maintenance of C. elegans. Celegans. 1999;2:51-67.

31. Porta-de-la-Riva M, Fontrodona L, Villanueva A, Cerón J. Basic Caenorhabditis elegans methods: Synchronization and observation. Journal of Visualized Experiments. 2012;(64):e4019. doi:10.3791/4019

32. Hulme SE, Shevkoplyas SS, Apfeld J, Fontana W, Whitesides GM. A microfabricated array of clamps for immobilizing and imaging C. elegans. Lab on a Chip. 2007;7(11):1515-1523. doi:10.1039/b707861g

33. Aryasomayajula A, Bayat P, Rezai P, Selvaganapathy PR. Microfluidic Devices and Their Applications. In: Springer Handbook of Nanotechnology. Springer; 2017:487-536.

34. Xia Y, Whitesides GM. Soft lithography. Annual Review of Materials Science. 1998;28(1):153-184. doi:10.1146/annurev.matsci.28.1.153

35. Youssef K, Archonta D, Kubiseski T, Tandon A, Rezai P. On-Demand Electric Field Induced Egg Laying of CAENORHABDITIS ELEGANS. In: Mtas 2019. Basel, Switzerland; 2019:392-393. 
1 36. Banse SA, Blue BW, Robinson KJ, Jarrett CM, Phillips PC. The Stress-Chip: A microfluidic platform

2 for stress analysis in Caenorhabditis elegans. Kim H, ed. PLOS ONE. 2019;14(5):e0216283.

3 doi:10.1371/journal.pone.0216283

4 37. Youssef K, Archonta D, Kubiseski T, Tandon A, Rezai P. Parallel-Channel Electrotaxis and Neuron

38. Ge A, Wang X, Ge M, et al. Profile analysis of: C. elegans rheotaxis behavior using a microfluidic

39. Schulz TJ, Zarse K, Voigt A, Urban N, Birringer M, Ristow M. Glucose Restriction Extends

40. Schlotterer A, Kukudov G, Bozorgmehr F, et al. C. elegans as model for the study of high glucose-

41. Wang X, Zhang L, Zhang L, et al. Effects of excess sugars and lipids on the growth and development of Caenorhabditis elegans. Genes and Nutrition. 2020;15(1):1. doi:10.1186/s12263-020-0659-1

42. Lee SJ, Murphy CT, Kenyon C. Glucose Shortens the Life Span of C. elegans by Downregulating

43. Alcántar-Fernández J, Navarro RE, Salazar-Martínez AM, Pérez-Andrade ME, Miranda-Ríos J.

45. GUZMAN AC DE, Kim EJ, Cho JH, Kim JH, Choi SS. High Glucose Diet Attenuates Dopaminergic

46. Lei W, Beaudoin-Chabot C, Thibault G. Glucose increases the lifespan of post-reproductive C. elegans

48. Schork NJ. Personalized medicine: time for one-person trials. Nature. 2015;520(7549):609-611. independently of FOXO. bioRxiv. June 2018:347435. doi:10.1101/347435 resolution. Microsystems \& Nanoengineering. 2018;4(1):6. doi:10.1038/s41378-018-0003-8 
bioRxiv preprint doi: https://doi.org/10.1101/2021.01.16.426931; this version posted January 18, 2021. The copyright holder for this preprint (which was not certified by peer review) is the author/funder. All rights reserved. No reuse allowed without permission.

1 50. Kopito RB, Levine E. Durable spatiotemporal surveillance of Caenorhabditis elegans response to 2 environmental cues. Lab on a Chip. 2014;14(4):764-770. doi:10.1039/c3lc51061a

3 